\title{
GRADIENT BOUNDS FOR SOLUTIONS OF ELLIPTIC AND PARABOLIC EQUATIONS
}

\author{
Vladimir I. Bogachev ${ }^{a}$, Giuseppe Da Prato ${ }^{b}$, Michael Röckner $^{c}$, \\ AND ZeEv SOBOL ${ }^{d}$ \\ ${ }^{a}$ : Department of Mechanics and Mathematics, Moscow State University, 119992 Moscow, \\ Russia \\ ${ }^{b}$ : Scuola Normale Superiore di Pisa, Piazza dei Cavalieri 7, I-56125 Pisa, Italy \\ c: Fakultät für Mathematik, Universität Bielefeld, D-33501 Bielefeld, Germany \\ ${ }^{d}$ : Department of Mathematics, University of Wales Swansea, Singleton Park, Swansea
} SA2 8PP, UK

\begin{abstract}
Let $L$ be a second order elliptic operator on $\mathbb{R}^{d}$ with a constant diffusion matrix and a dissipative (in a weak sense) drift $b \in L_{l o c}^{p}$ with some $p>d$. We assume that $L$ possesses a Lyapunov function, but no local boundedness of $b$ is assumed. It is known that then there exists a unique probability measure $\mu$ satisfying the equation $L^{*} \mu=0$ and that the closure of $L$ in $L^{1}(\mu)$ generates a Markov semigroup $\left\{T_{t}\right\}_{t \geq 0}$ with the resolvent $\left\{G_{\lambda}\right\}_{\lambda>0}$. We prove that, for any Lipschitzian function $f \in L^{1}(\mu)$ and all $t, \lambda>0$, the functions $T_{t} f$ and $G_{\lambda} f$ are Lipschitzian and

$$
\left|\nabla T_{t} f(x)\right| \leq T_{t}|\nabla f|(x) \text { and }\left|\nabla G_{\lambda} f(x)\right| \leq \frac{1}{\lambda} G_{\lambda}|\nabla f|(x) .
$$
\end{abstract}

An analogous result is proved in the parabolic case.

Suppose that for every $t \in[0,1]$, we are given a a strictly positive definite symmetric matrix $A(t)=\left(a^{i j}(t)\right)$ and a measurable vector field $x \mapsto b(t, x)=$ $\left(b^{1}(t, x), \ldots, b^{n}(t, x)\right)$.

Let $L_{t}$ be the elliptic operator on $\mathbb{R}^{d}$ given by

$$
L_{t} u(x)=\sum_{i, j \leq d} a^{i j}(t, x) \partial_{x_{i}} \partial_{x_{j}} u(x)+\sum_{i \leq d} b^{i}(t, x) \partial_{x_{i}} u(x) .
$$

Suppose that $A$ and $b$ satisfy the following hypotheses:

(Ha) $\sup _{t \in[0,1]}\left(\|A(t)\|+\left\|A(t)^{-1}\right\|\right)<\infty, \sup _{t \in[0,1]}\|b(t, \cdot)\|_{L^{p}(U)}<\infty$ for every ball $U$ in $\mathbb{R}^{d}$ with some $p>d, p \geq 2$.

$(\mathrm{Hb}) b$ is dissipative in the following sense: for every $t \in[0,1]$ and every $h \in \mathbb{R}^{d}$, there exists a measure zero set $N_{t, h} \subset \mathbb{R}^{d}$ such that

$$
(b(t, x+h)-b(t, x), h) \leq 0 \quad \text { for all } x \in \mathbb{R}^{d} \backslash N_{t, h} .
$$

(Hc) for every $t \in[0,1]$, there exists a Lyapunov function $V_{t}$ for $L_{t}$, i.e., a nonnegative $C^{2}$-function $V_{t}$ such that $V_{t}(x) \rightarrow+\infty$ and $L_{t} V_{t}(x) \rightarrow-\infty$ as $|x| \rightarrow \infty$.

We consider the parabolic equation

$$
\frac{\partial u}{\partial t}=L_{t} u, \quad u(0, x)=f(x),
$$


where $f$ is a bounded Lipschitz function. A locally integrable function $u$ on $[0,1] \times \mathbb{R}^{d}$ is called a solution if, for every $t \in(0,1]$, one has $u(t, \cdot) \in W_{\text {loc }}^{1,2}\left(\mathbb{R}^{d}\right)$, the functions $\partial_{x_{i}} \partial_{x_{j}} u$ and $b^{i} \partial_{x_{i}} u$ are integrable on the sets $[0,1] \times K$ for every cube $K$ in $\mathbb{R}^{d}$, and for every $\varphi \in C_{0}^{\infty}\left(\mathbb{R}^{d}\right)$ and all $t \in[0,1]$ one has

$$
\int_{\mathbb{R}^{d}} u(t, x) \varphi(x) d x=\int_{\mathbb{R}^{d}} f(x) \varphi(x) d x+\int_{0}^{t} \int_{\mathbb{R}^{d}} L_{s} \varphi(x) u(s, x) d x d s .
$$

In the case where $A$ and $b$ are independent of $t$, so that we have a single operator $L$, Hypotheses (Ha) and (Hc) imply (see [6] and [8]) that there exists a unique probability measure $\mu$ on $\mathbb{R}^{d}$ such that $\mu$ has a strictly positive continuous weakly differentiable density $\varrho,|\nabla \varrho| \in L_{l o c}^{p}\left(\mathbb{R}^{d}\right)$, and $L^{*} \mu=0$ in the following weak sense:

$$
\int L u d \mu=0 \quad \text { for all } u \in C_{0}^{\infty}\left(\mathbb{R}^{d}\right)
$$

The closure $\bar{L}$ of $L$ with domain $C_{0}^{\infty}\left(\mathbb{R}^{d}\right)$ in $L^{1}(\mu)$ generates a Markov semigroup $\left\{T_{t}\right\}_{t>0}$ for which $\mu$ is invariant. Let $D(\bar{L})$ denote the domain of $\bar{L}$ in $L^{1}(\mu)$ and let $\left\{G_{\lambda}\right\}_{\lambda>0}$ denote the corresponding resolvent, i.e., $G_{\lambda}=(\lambda-\bar{L})^{-1}$. The restrictions of $T_{t}$ and $G_{\lambda}$ to $L^{2}(\mu)$ are contractions on $L^{2}(\mu)$. In particular, if $v \in D(\bar{L})$ is such that $\lambda v-\bar{L} v=g \in L^{2}(\mu)$, then $v \in L^{2}(\mu)$. Moreover, it follows by [8, Theorem 2.8] that one has $v \in H_{l o c}^{2,2}\left(\mathbb{R}^{d}\right)$ and $\bar{L} v=L v$ a.e., so that one has a.e.

$$
\lambda v-L v=g .
$$

In fact, due to our assumptions on the coefficients of $L$ one has even $v \in$ $W_{l o c}^{p, 2}\left(\mathbb{R}^{d}\right)$ (see [10]). It has been shown in [3] that for every function $f \in L^{1}(\mu)$ that is Lipschitzian with constant $C$ and all $t, \lambda>0$, the continuous version of the function $T_{t} f$ is Lipschitzian with constant $C$, and the continuous version of $G_{\lambda} f$ is Lipschitzian with constant $\lambda^{-1} C$. Here we establish pointwise estimates in both cases and prove their parabolic analogue. The main results of this work are the following two theorems.

Theorem 1. Suppose that $A$ and $b$ are independent of t and satisfy (Ha), (Hb) and $(\mathrm{Hc})$. Then, for any Lipschitzian function $f \in L^{1}(\mu)$ and all $t, \lambda>0, T_{t} f$ and $G_{\lambda} f$ have Lipschitzian versions such that

$$
\left|\nabla T_{t} f(x)\right| \leq T_{t}|\nabla f|(x) \quad \text { and } \quad\left|\nabla G_{\lambda} f(x)\right| \leq \frac{1}{\lambda} G_{\lambda}|\nabla f|(x)
$$

for the corresponding continuous versions. In particular,

$$
\sup _{x, t}\left|\nabla T_{t} f(x)\right| \leq \sup _{x}|\nabla f(x)|, \quad \sup _{x}\left|\nabla G_{\lambda} f(x)\right| \leq \frac{1}{\lambda} \sup _{x}|\nabla f(x)| .
$$

Theorem 2. Suppose that $A$ and $b$ satisfy (Ha), (Hb) and (Hc). Then, for any bounded Lipschitzian function $f$ there is a solution $u$ of equation (2) such that for all t one has

$$
\sup _{x}|\nabla u(t, x)| \leq \sup _{x}|\nabla f(x)| .
$$


In the case where $A=I$ and $b=0$, estimate (6) has been established in [12], [13] for solutions of boundary value problems in bounded domains. It should be noted that gradient estimates of the type

$$
\sup _{x}|\nabla u(x, t)| \leq C(t) \sup _{x}|f(x)|
$$

for solutions of parabolic equations have been obtained by many authors, see, e.g., [1], [2], [11], [15], and the references therein. Such estimates do not require $(\mathrm{Hb})$ and one has $C(t) \rightarrow+\infty$ as $t \rightarrow 0$ or $t \rightarrow+\infty$. In contrast to this type of estimates, our theorems mean a contraction property on Lipschitz functions rather than a smoothing property. It is likely that some results of the cited works, established for sufficiently regular $b$, can be extended to more general drifts satisfying just $(\mathrm{Ha})$, but not $(\mathrm{Hb})$.

A short proof of the following result can be found in [3].

Lemma 1. Suppose that $b$ is infinitely differentiable, Lipschitzian, and strongly dissipative, so for some $\alpha>0$, one has

$$
(b(x+h)-b(x), h) \leq-\alpha(h, h) \quad \text { for all } x, h \in \mathbb{R}^{d} .
$$

Then, for any $\lambda>0$ and any smooth bounded Lipschitzian function $f$, one has pointwise

$$
\left|\nabla G_{\lambda} f\right| \leq G_{\lambda}|\nabla f|
$$

In particular, $\sup _{x}\left|\nabla G_{\lambda} f(x)\right| \leq \lambda^{-1} \sup _{x}|\nabla f(x)|$.

Proof of Theorem 1. The estimate with the suprema has been proven in [3], and the stronger pointwise estimate can be derived from that proof. For the reader's convenience, instead of recursions to the steps of the proof in [3] we reproduce the whole proof and explain why it yields a stronger conclusion. We recall that if a sequence of functions on $\mathbb{R}^{d}$ is uniformly Lipschitzian with constant $L$ and bounded at a point, then it contains a subsequence that converges uniformly on every ball to a function that is Lipschitzian with the same constant. Therefore, approximating $f$ in $L^{1}(\mu)$ by a sequence of bounded smooth functions $f_{j}$ with

$$
\sup _{x}\left|\nabla f_{j}(x)\right| \leq \sup _{x}|\nabla f(x)|,
$$

it suffices to prove (5) for smooth bounded $f$. Moreover, due to Euler's formula $T_{t} f=\lim _{n}\left(\frac{t}{n} G_{\frac{t}{n}}\right)^{n} f$, it suffices to establish the resolvent estimate. First we construct a suitable sequence of smooth strongly dissipative Lipschitzian vector fields $b_{k}$ such that $b_{k} \rightarrow b$ in $L^{p}\left(U, \mathbb{R}^{d}\right)$ for every ball $U$ as $k \rightarrow \infty$. Let $\sigma_{j}(x)=j^{-d} \sigma(x / j)$, where $\sigma$ is a smooth compactly supported probability density. Let $\beta_{j}:=b * \sigma_{j}$. Then $\beta_{j}$ is smooth and dissipative and $\beta_{j} \rightarrow b$, $j \rightarrow \infty$, in $L^{p}\left(U, \mathbb{R}^{d}\right)$ for every ball $U$. For every $\alpha>0$, the mapping $I-\alpha \beta_{j}$ is a homeomorphism of $\mathbb{R}^{d}$ and the inverse mapping $\left(I-\alpha \beta_{j}\right)^{-1}$ is Lipschitzian with constant $\alpha^{-1}$ (see [9]). Let us consider the Yosida approximations

$$
F_{\alpha}\left(\beta_{j}\right):=\alpha^{-1}\left(\left(I-\alpha \beta_{j}\right)^{-1}-I\right)=\beta_{j} \circ\left(I-\alpha \beta_{j}\right)^{-1} .
$$


It is known (see $\left[9, \mathrm{Ch}\right.$. II]) that $\left|F_{\alpha}\left(\beta_{j}\right)(x)\right| \leq\left|\beta_{j}(x)\right|$, the mappings $F_{\alpha}\left(\beta_{j}\right)$ converge locally uniformly to $\beta_{j}$ as $\alpha \rightarrow 0$, and one has

$$
\left(F_{\alpha}\left(\beta_{j}\right)(x)-F_{\alpha}\left(\beta_{j}\right)(y), x-y\right) \leq 0 .
$$

Thus, the sequence $b_{k}:=F_{\frac{1}{k}}\left(b * \sigma_{k}\right)-\frac{1}{k} I, k \in \mathbb{N}$, is the desired one. For every $k \in \mathbb{N}$, let $L_{k}$ be the elliptic operator defined by (1) with the same constant matrix $A$ and drift $b_{k}$ in place of $b$. Let $\mu_{k}=\varrho_{k} d x$ be the corresponding invariant probability measure and let $G_{\lambda}^{(k)}$ denote the associated resolvent family on $L^{1}\left(\mu_{k}\right)$. Since $b_{k}$ is smooth, Lipschitzian and strongly dissipative, we obtain that $v_{k}:=G_{\lambda}^{(k)} f$ is smooth, bounded, Lipschitzian and

$$
\sup _{x}\left|v_{k}(x)\right| \leq \frac{1}{\lambda} \sup _{x}|f(x)| \text { and } \sup _{x}\left|\nabla v_{k}(x)\right| \leq \frac{1}{\lambda} \sup _{x}|\nabla f(x)|
$$

by the lemma. Moreover, for every ball $U \subset \mathbb{R}^{d}$, the functions $v_{k}$ are uniformly bounded in the Sobolev space $W^{2,2}(U)$, since the mappings $\left|b_{k}\right|$ are bounded in $L^{p}(U)$ uniformly in $k$ and $f$ is bounded. This follows from the fact that for any solution $w \in W^{2,2}(U)$ of the equation

$$
\sum_{i, j \leq d} a^{i j} \partial_{x_{i}} \partial_{x_{i}} w+\sum_{i \leq d} b^{i} \partial_{x_{i}} \partial_{x_{i}} w-\lambda w=g
$$

one has $\|w\|_{W^{2,2}(U)} \leq C\|w\|_{L^{2}(U)}$, where $C$ is a constant that depends on $U, A$, and the quantity $\kappa:=\|g\|_{L^{2}(U)}+\||b|\|_{L^{p}(U)}$ in such a way that as a function of $\kappa$ it is locally bounded. Thus, the sequence $\left\{v_{k}\right\}$ contains a subsequence, again denoted by $\left\{v_{k}\right\}$, that converges locally uniformly to a bounded Lipschitzian function $v \in W_{l o c}^{2,2}\left(\mathbb{R}^{d}\right)$ such that

$$
\sup _{x}|v(x)| \leq \lambda^{-1} \sup _{x}|f(x)| \text { and } \sup _{x}|\nabla v(x)| \leq \lambda^{-1} \sup _{x}|\nabla f(x)|,
$$

and, in addition, the restrictions of $v_{k}$ to any ball $U$ converge to $\left.v\right|_{U}$ weakly in $W^{2,2}(U)$.

Let $\widehat{L}$ be the elliptic operator with the same second order part as $L$, but with drift is $\widehat{b}=2 A \nabla \varrho / \varrho-b$. Then by the integration by parts formula

$$
\int \psi L \varphi d \mu=\int \varphi \widehat{L} \psi d \mu \text { for all } \psi, \varphi \in C_{0}^{\infty}\left(\mathbb{R}^{d}\right) .
$$

In addition, for any $\lambda>0$, the ranges of $\lambda-L$ and $\lambda-\widehat{L}$ on $C_{0}^{\infty}\left(\mathbb{R}^{d}\right)$ are dense in $L^{1}(\mu)$. The operator $\widehat{L}$ also generates a Markov semigroup on $L^{1}(\mu)$ with respect to which $\mu$ is invariant. The corresponding resolvent is denoted by $\widehat{G}_{\lambda}$. For the proofs we refer to [7, Proposition 2.9] or [14, Proposition 1.10(b)] (see also [8, Theorem 3.1]).

Now we show that $v=G_{\lambda} f$. Note that $\varrho_{k} \rightarrow \varrho$ uniformly on balls according to [6], [5]. Hence, given $\varphi \in C_{0}^{\infty}\left(\mathbb{R}^{d}\right)$ with support in a ball $U$, we have

$$
\int[\lambda v-L v-f] \varphi \varrho d x=\lim _{k \rightarrow \infty} \int\left[\lambda v_{k}-L_{k} v_{k}-f\right] \varphi \varrho_{k} d x=0
$$


by weak convergence of $v_{k}$ to $v$ in $W^{2,2}(U)$ combined with convergence of $b_{k}$ to $b$ in $L^{p}\left(U, \mathbb{R}^{d}\right)$. Therefore, by the integration by parts formula

$$
\int v(\lambda \varphi-\widehat{L} \varphi) d \mu=\int f \varphi d \mu
$$

for all $\varphi \in C_{0}^{\infty}\left(\mathbb{R}^{d}\right)$. The function $G_{\lambda} f$ is bounded and satisfies the same relation, so it remains to recall that if a bounded function $u$ satisfies the equality

$$
\int u(\lambda \varphi-\widehat{L} \varphi) d \mu=0
$$

for all $\varphi \in C_{0}^{\infty}\left(\mathbb{R}^{d}\right)$, then $u=0$ a.e., since $(\lambda-\widehat{L})\left(C_{0}^{\infty}\left(\mathbb{R}^{d}\right)\right)$ is dense in $L^{1}(\mu)$.

Now we turn to the pointwise estimate $\left|\nabla G_{\lambda} f(x)\right| \leq \lambda^{-1} G_{\lambda}|\nabla f|(x)$. Suppose first that $f \in C_{0}^{\infty}\left(\mathbb{R}^{d}\right)$. The desired estimate holds for every $G_{\lambda}^{(k)}$ in place of $G_{\lambda}$. It has been shown above that $v=G_{\lambda} f$ is a weak limit of $v_{k}=G_{\lambda}^{(k)} f$ in $W^{2,2}(U)$ for every ball $U$. In addition, the functions $G_{\lambda}^{(k)}|\nabla f|$ converge weakly in $W^{2,2}(U)$ to the function $G_{\lambda}|\nabla f|$, which is also clear by the above reasoning. Since the embedding of $W^{2,2}(U)$ into $W^{2,1}(U)$ is compact, we may assume, passing to a subsequence, that $\nabla G_{\lambda}^{(k)} f(x) \rightarrow \nabla G_{\lambda} f(x)$ and $G_{\lambda}^{(k)}|\nabla f|(x) \rightarrow G_{\lambda}|\nabla f|(x)$ almost everywhere on $U$. Hence we arrive at the desired estimate. If $f$ is Lipschitzian and has bounded support, we can find uniformly Lipschitzian functions $f_{n} \in C_{0}^{\infty}\left(\mathbb{R}^{d}\right)$ vanishing outside some ball such that $f_{n} \rightarrow f$ uniformly and $\nabla f_{n} \rightarrow \nabla f$ a.e. Then, by the same reasons as above, one has $G_{\lambda}\left|\nabla f_{n}\right| \rightarrow G_{\lambda}|\nabla f|$ and $\nabla G_{\lambda} f_{n} \rightarrow \nabla G_{\lambda} f$ in $L^{2}(U)$. Passing to an almost everywhere convergent subsequence we obtain a pointwise inequality. Finally, in the case of a general Lipschitzian function $f \in L^{1}(\mu)$, we can find uniformly Lipschitzian functions $\zeta_{n}$ such that $0 \leq \zeta_{n} \leq 1$ and $\zeta_{n}(x)=1$ if $|x| \leq n$. Let $f_{n}=f \zeta_{n}$. By the previous step we have

$$
\left|\nabla G_{\lambda} f_{n}(x)\right| \leq \lambda^{-1} G_{\lambda}\left|\nabla f_{n}\right|(x) .
$$

The functions $f_{n}$ are uniformly Lipschitzian. Hence, for every ball $U$, the sequence of functions $\left.G_{\lambda} f_{n}\right|_{U}$ is bounded in the norm of $W^{2,2}(U)$. In addition, the functions $G_{\lambda}\left|\nabla f_{n}\right|$ on $U$ converge to $G_{\lambda}|\nabla f|$ in $L^{2}(U)$, since $\left|\nabla f_{n}\right| \rightarrow|\nabla f|$ in $L^{2}(\mu)$ by the Lebesgue dominated convergence theorem. Therefore, the same reasoning as above completes the proof.

Proof of Theorem 2. Suppose first that $A$ is piece-wise constant, i.e., there exist finitely many intervals $\left[0, t_{1}\right),\left[t_{1}, t_{2}\right), \ldots,\left[t_{n}, 1\right]$ such that $A(t)=A_{k}$ whenever $t_{k-1} \leq t<t_{k}$, where each $A_{k}$ is a strictly positive symmetric matrix. In addition, let us assume that there exist vector fields $b_{k}$ such that $b(t, x)=b_{k}(x)$ whenever $t_{k-1} \leq t<t_{k}$. Then we obtain a solution $u$ by successively applying the semigroups $T_{t}^{(k)}$ generated by the elliptic operators with the diffusion matrices $A_{k}$ and drifts $b_{k}$, i.e.,

$$
u(t, x)=T_{t-t_{k-1}} T_{t_{k-1}} \cdots T_{t_{1}} f(x) \quad \text { whenever } t \in\left[t_{k-1}, t_{k}\right) .
$$


The conclusion of Theorem 2 in this case follows by Theorem 1. Our next step is to approximate $A$ and $b$ by mappings of the above form in such a way that the corresponding sequence of solutions would converge to a solution of our equation. Let us observe that, for an arbitrary sequence of such solutions $u_{k}$ corresponding to piece-wise constant in time coefficients, for every compactly supported function $\varphi$ on $\mathbb{R}^{d}$, the functions

$$
t \mapsto \int_{\mathbb{R}^{d}} \varphi(x) u_{k}(t, x) d x
$$

are uniformly Lipschitzian provided that the operator norms of the matrix functions $A_{k}$ are uniformly bounded and that the $L^{p}(K)$-norms of the vector fields $b_{k}(t, \cdot)$ are uniformly bounded for every fixed cube $K$ in $\mathbb{R}^{d}$. This is clear, because (2) can be written as

$$
\int_{\mathbb{R}^{d}} \varphi(x) u(t, x) d x=\int_{0}^{t} \int_{\mathbb{R}^{d}}\left[L_{s} \varphi(x) u(s, x)+\varphi(x) b^{i}(s, x) \partial_{x_{i}} u(s, x)\right] d x d s,
$$

where in the case $u=u_{k}$ we have

$$
|u(s, x)| \leq \sup |f(x)| \text { and }\left|\nabla_{x} u(s, x)\right| \leq \sup |\nabla f(x)| .
$$

One can choose a subsequence in $\left\{u_{k}\right\}$ that converges to some function $u$ on $[0,1] \times \mathbb{R}^{d}$ in the following sense: for every cube $K$ in $\mathbb{R}^{d}$, the functions the restrictions of the functions $u_{k}$ to $[0,1] \times K$ converge weakly to $u$ in the space $L^{2}\left([0,1], W^{2,2}(K)\right)$, where each $u_{k}$ is regarded as a mapping $t \mapsto u_{k}(t, \cdot)$ from $[0,1]$ to $W^{2,2}(K)$. Passing to another subsequence we obtain

$$
\lim _{n \rightarrow \infty} \int_{\mathbb{R}^{d}} \varphi(x) u_{k}(t, x) d x=\int_{\mathbb{R}^{d}} \varphi(x) u(t, x) d x
$$

for all $t \in[0,1]$ and all smooth compactly supported $\varphi$. Indeed, for a given function $\varphi$ this is possible due to the uniform Lipschitzness of the functions (7). Then our claim is true for a countable family of functions $\varphi$, which, on account of the uniform boundedness of $u_{k}$, yields the claim for all $\varphi$. Therefore, it remains to find approximations $A_{k}$ and $b_{k}$ such that, for every function $\psi \in$ $C[0,1]$, the integrals

$$
\int_{0}^{1} \psi(s) \int_{\mathbb{R}^{d}}\left[L_{s}^{(k)} \varphi(x) u_{k}(s, x)+\varphi(x) b_{k}^{i}(s, x) \partial_{x_{i}} u_{k}(s, x)\right] d x d s
$$

would converge to the corresponding integral with $A, b$, and $u$. Clearly, it suffices to obtain the desired convergence for suitable countable families of functions $\varphi_{i}$ and $\psi_{j}$. Let us fix two sequences $\left\{\psi_{j}\right\} \subset C[0,1]$ and $\left\{\varphi_{i}\right\} \subset C_{0}^{\infty}\left(\mathbb{R}^{d}\right)$ with the following property: every compactly supported square-integrable function $v$ on $[0,1] \times \mathbb{R}^{d}$ can be approximated in $L^{2}$ by a sequence of finite linear combinations of products $\psi_{j} \varphi_{i}$. Let us consider the functions

$$
\alpha_{i, j, k}(t):=a^{i j}(t) \psi_{k}(t), \quad \beta_{i, j, k}(t):=\psi_{k}(t) \int_{\mathbb{R}^{d}} b^{i}(s, x) \varphi_{j}(x) d x,
$$




$$
\theta_{k, i}(t)=\int_{[-k, k]^{d}} b_{i}(t, x)^{2} d x
$$

Let $\mathcal{F}$ denote the obtained countable family of functions extended periodically from $[0,1)$ to $\mathbb{R}$ with period 1 . It is well known that, for almost every $s \in$ $[0,1)$, the Riemannian sums $R_{n}(\theta)(s)=2^{-n} \sum_{k=1}^{2^{n}} \theta\left(s+k 2^{-n}\right)$ converge to the integral of $\theta$ over $[0,1]$ for each $\theta \in \mathcal{F}$. It follows that one can find points $t_{n, l}$, $l=1, \ldots, N_{n}, n \in \mathbb{N}$, such that

$$
0=t_{n, 0}<t_{n, 1}<t_{n, 2}<\cdots<t_{n, N_{n}}=1
$$

and, for every $\theta \in \mathcal{F}$, letting $\theta_{n}(t):=\theta\left(t_{n, l}\right)$ whenever $t_{n, l-1} \leq t<t_{n, l}$, one has

$$
\int_{0}^{1} \theta_{n}(t) d t \rightarrow \int_{0}^{1} \theta(t) d t
$$

To this end, we pick a common point $s_{0}$ of convergence of the Riemann sums $R_{n}(\theta)\left(s_{0}\right)$ to the respective integrals and let $t_{n, l}=s_{0}+l 2^{-n}(\bmod 1)$. By using the points $t_{n, l}$, one obtains the desired piece-wise constant approximations of $A$ and $b$. Namely, let $A_{n}(t)=A\left(t_{n, l}\right)$ and $b_{n}(t, x)=b\left(t_{n, l}, x\right)$ whenever $t_{n, l-1} \leq t<t_{n, l}$. As explained above, passing to a subsequence, we may assume that the corresponding solutions $u_{n}$ converge to a function $u$ such that, for every cube $K=[-m, m]^{d}$ in $\mathbb{R}^{d}$ and every $t \in(0,1]$, one has

$$
\left.u(t, \cdot)\right|_{K} \in W^{2,2}(K), \quad \int_{0}^{1}\|u(t, \cdot)\|_{W^{2,2}(K)}^{2} d t<\infty,
$$

and for any function $\zeta \in L^{2}([0,1] \times K)$ there holds the equalities

$$
\begin{aligned}
\lim _{n \rightarrow \infty} \int_{0}^{1} \int_{K} \zeta(t, x) u_{n}(t, x) d x d t & =\int_{0}^{1} \int_{K} \zeta(t, x) u(t, x) d x d t \\
\lim _{n \rightarrow \infty} \int_{0}^{1} \int_{K} \zeta(t, x) \partial_{x_{i}} \partial_{x_{j}} u_{n}(t, x) d x d t & =\int_{0}^{1} \int_{K} \zeta(t, x) \partial_{x_{i}} \partial_{x_{j}} u(t, x) d x d t \\
\lim _{n \rightarrow \infty} \int_{0}^{1} \int_{K} \zeta(t, x) \partial_{x_{i}} u_{n}(t, x) d x d t & =\int_{0}^{1} \int_{K} \zeta(t, x) \partial_{x_{i}} u(t, x) d x d t \\
\lim _{n \rightarrow \infty} \int_{0}^{1} \int_{K} b_{i}^{n}(t, x)^{2} d x d t & =\int_{0}^{1} \int_{K} b_{i}(t, x)^{2} d x d t
\end{aligned}
$$

Note that for any cube $K \subset \mathbb{R}^{d}$, the restrictions of the functions $b_{n}^{i}$ to $[0,1] \times K$ converge to the restriction of $b^{i}$ in the norm of $L^{2}([0,1] \times K)$. This is clear from the last displayed equality, which gives convergence of $L^{2}$-norms, along with convergence of the Riemann sums $R_{n}\left(\beta_{i, j, k}\right)\left(s_{0}\right)$ to the integral of $\beta_{i, j, k}$ over $[0,1]$, which yields weak convergence (we recall that if a sequence of vectors $h_{n}$ in a Hilbert space $H$ converges weakly to a vector $h$ and the norms of $h_{n}$ converge to the norm of $h$, then there is norm convergence). It follows that for 
any $\psi \in C[0,1]$ and any $\varphi \in C_{0}^{\infty}\left(\mathbb{R}^{d}\right)$ with support in $[-m, m]^{d}$, we have

$$
\begin{aligned}
\lim _{n \rightarrow \infty} \int_{0}^{1} \psi(t) a_{n}^{i j}(t) \int_{\mathbb{R}^{d}} \partial_{x_{i}} \partial_{x_{j}} \varphi(x) & u_{n}(t, x) d x d t \\
= & \int_{0}^{1} \psi(t) a^{i j}(t) \int_{\mathbb{R}^{d}} \partial_{x_{i}} \partial_{x_{j}} \varphi(x) u(t, x) d x d t .
\end{aligned}
$$

In addition,

$$
\begin{aligned}
& \lim _{n \rightarrow \infty} \int_{0}^{1} \psi(t) \int_{\mathbb{R}^{d}} \varphi(x) \partial_{x_{i}} u_{n}(t, x) b_{n}^{i}(t, x) d x d t \\
&=\int_{0}^{1} \psi(t) \int_{\mathbb{R}^{d}} \varphi(x) \partial_{x_{i}} u(t, x) b^{i}(t, x) d x d t .
\end{aligned}
$$

This follows by norm convergence of $b_{n}^{i}$ to $b^{i}$ and weak convergence of $\varphi \partial_{x_{i}} u_{n}$ to $\varphi \partial_{x_{i}} u$ in $L^{2}\left([0,1] \times[-m, m]^{d}\right)$. Therefore, for every $\varphi \in C_{0}^{\infty}\left(\mathbb{R}^{d}\right)$, one has

$$
\int_{\mathbb{R}^{d}} \varphi(x) u(t, x) d x d t=\int_{\mathbb{R}^{d}} \varphi(x) f(x) d x+\int_{0}^{t} \int_{\mathbb{R}^{d}} \varphi(x) L_{t} u(t, x) d x d t
$$

for almost all $t \in[0,1]$, since the integrals of both sides multiplied by any function $\psi \in C_{0}^{\infty}(0,1)$ coincide. Taking into account the continuity of both sides (the left-hand side is Lipschitzian as explained above), we conclude that the equality holds for all $t \in[0,1]$.

Acknowledgements. This work has been supported in part by the RFBR project 04-01-00748, the DFG Grant 436 RUS 113/343/0(R), the INTAS project 0351-5018, the Scientific Schools Grant 1758.2003.1, the DFG-Forschergruppe "Spectral Analysis, Asymptotic Distributions, and Stochastic Dynamics", the BiBoSreseach centre, and the research programme "Analisi e controllo di equazioni di evoluzione deterministiche e stocastiche" from the Italian "Ministero della Ricerca Scientifica e Tecnologica".

\section{REFERENCES}

[1] Bertoldi M., Fornato S. Gradient estimates in parabolic problems with unbounded coefficients. Preprint del Dipartimento di Matematica, Universitá di Parma, no. 316, 2003.

[2] Bertoldi M., Fornato S., Lorenzi L. Gradient estimates for parabolic problems with unbounded coefficients in non convex domains. Tübinger Berichte zur Funkt. Analysis. H. 13. 2003-2004. S. 14-45.

[3] Bogachev V.I., Da Prato G., Röckner M., Sobol Z. Global gradient bounds for dissipative diffusion operators. C. R. Acad. Sci. Paris, sér. I, 339 (2004), 277-282.

[4] Bogachev V.I., Krylov N.V., Röckner M. Regularity of invariant measures: the case of non-constant diffusion part. J. Funct. Anal. 138 (1996), no. 1, 223-242.

[5] Bogachev V.I., Krylov N.V., Röckner M. On regularity of transition probabilities and invariant measures of singular diffusions under minimal conditions. Comm. Partial Diff. Equations 26 (2001), no. 11-12, 2037-2080.

[6] Bogachev V.I., Röckner M. A generalization of Hasminskii's theorem on existence of invariant measures for locally integrable drifts. Theory Probab. Appl. 45 (2000), no. 3, $417-436$. 
[7] Bogachev V.I., Röckner M., Stannat W. Uniqueness of invariant measures and maximal dissipativity of diffusion operators on $L^{1}$. In: Infinite dimensional stochastic analysis, pp. 39-54, P. Clement et al. eds. Royal Netherlands Academy of Arts and Sciences, Amsterdam, 2000.

[8] Bogachev V.I., Röckner M., Stannat W. Uniqueness of solutions of elliptic equations and uniqueness of invariant measures of diffusions. Sbornik Math. 193 (2002), no. 7, 945-976.

[9] Brézis H. Opérateurs maximaux monotones et semi-groupes de contractions dans les espaces de Hilbert. North-Holland, Amsterdam - London; American Elsevier, New York, 1973.

[10] Chicco M. Solvability of the Dirichlet problem in $H^{2, p}(\Omega)$ for a class of linear second order elliptic partial differential equations. Boll. Un. Mat. Ital. (4) 4 (1971), 374-387.

[11] Fornaro S., Metafune G., Priola E. Gradient estimates for Dirichlet parabolic problems in unbounded domains. J. Diff. Eq. 205 (2004), 329-353.

[12] Kahane C.S. A gradient estimate for solutions of the heat equation. Czechoslovak Math. J. 48(123) (1998), no. 4, 711-725.

[13] Kahane C.S. A gradient estimate for solutions of the heat equation. II. Czechoslovak Math. J. 51(126) (2001), no. 1, 39-44.

[14] Stannat W. (Nonsymmetric) Dirichlet operators on $L^{1}$ : existence, uniqueness and associated Markov processes. Ann. Scuola Norm. Sup. Pisa Cl. Sci. (4) 28 (1999), no. 1, 99-140.

[15] Wang F.-Y. Gradient estimates of Dirichlet heat semigroups and application to isoperimetric inequalities. Ann. Probab. 32 (2004), no. 1A, 424-440. 